\title{
Piezoelectric graphene field effect transistor pressure sensors for tactile sensing
}

N. Yogeswaran, W. T. Navaraj, S. Gupta, F. Liu, V. Vinciguerra, L. Lorenzelli, and R. Dahiya

Citation: Appl. Phys. Lett. 113, 014102 (2018); doi: 10.1063/1.5030545

View online: https://doi.org/10.1063/1.5030545

View Table of Contents: http://aip.scitation.org/toc/apl/113/1

Published by the American Institute of Physics

\section{Articles you may be interested in}

Re doping induced $2 \mathrm{H}-1 \mathrm{~T}$ phase transformation and ferromagnetism in $\mathrm{MoS}_{2}$ nanosheets

Applied Physics Letters 113, 013101 (2018); 10.1063/1.5027535

Acoustic wave sparsely activated localization microscopy (AWSALM): Super-resolution ultrasound imaging using acoustic activation and deactivation of nanodroplets

Applied Physics Letters 113, 014101 (2018); 10.1063/1.5029874

Local structures and temperature-driven polarization rotation in $\mathrm{Zr}$-rich $\mathrm{PbZr}_{1-\mathrm{x}} \mathrm{Ti}_{\mathrm{x}} \mathrm{O}_{3}$

Applied Physics Letters 113, 012901 (2018); 10.1063/1.5024422

Enhanced n-doping of epitaxial graphene on $\mathrm{SiC}$ by bismuth

Applied Physics Letters 113, 011602 (2018); 10.1063/1.5029541

Hybrid energy harvesting from mechanical vibrations and magnetic field

Applied Physics Letters 113, 013901 (2018); 10.1063/1.5038412

Exploration of exciton behavior in atomically thin $\mathrm{WS}_{2}$ layers by ionic gating

Applied Physics Letters 113, 013104 (2018); 10.1063/1.5022327

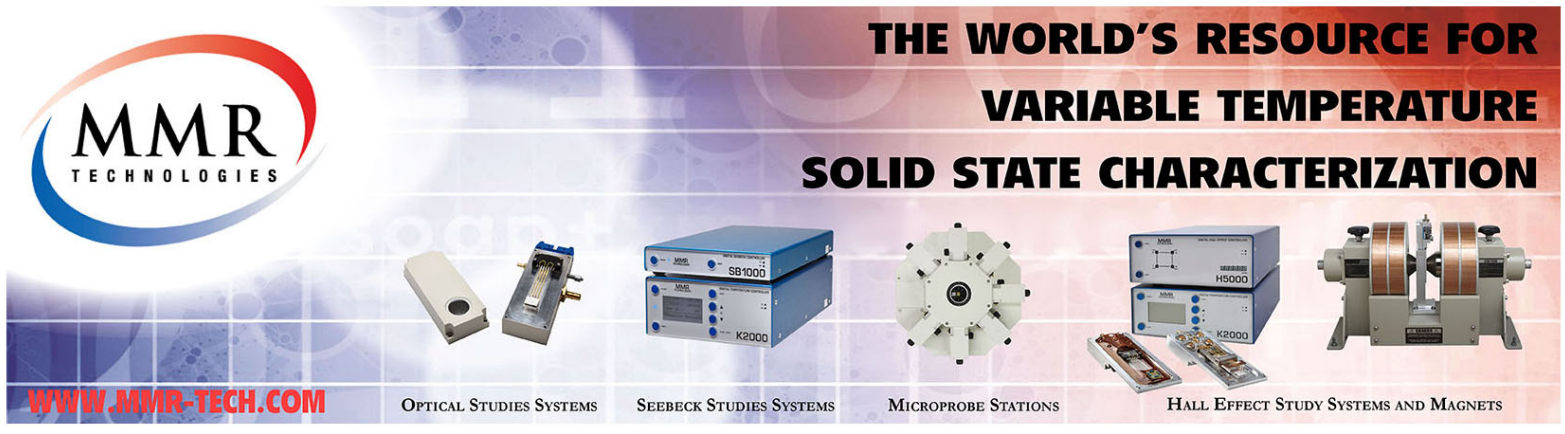




\title{
Piezoelectric graphene field effect transistor pressure sensors for tactile sensing
}

\author{
N. Yogeswaran, ${ }^{1}$ W. T. Navaraj, ${ }^{1}$ S. Gupta, ${ }^{1}$ F. Liu, ${ }^{1}$ V. Vinciguerra,${ }^{2}$ L. Lorenzelli, ${ }^{3}$ \\ and R. Dahiya ${ }^{1}$ \\ ${ }^{1}$ Bendable Electronics and Sensing Technologies (BEST) Group, School of Engineering, \\ University of Glasgow, Glasgow G12 8QQ, United Kingdom \\ ${ }^{2}$ ST Microelectronics, Catania 95121, Italy \\ ${ }^{3}$ Microsystems Technology (MST) Group, Fondazione Bruno Kessler, Trento 38123, Italy
}

(Received 22 March 2018; accepted 18 June 2018; published online 2 July 2018)

\begin{abstract}
This paper presents graphene field-effect transistor (GFET) based pressure sensors for tactile sensing. The sensing device comprises GFET connected with a piezoelectric metal-insulator-metal (MIM) capacitor in an extended gate configuration. The application of pressure on MIM generates a piezo-potential which modulates the channel current of GFET. The fabricated pressure sensor was tested over a range of $23.54-94.18 \mathrm{kPa}$, and it exhibits a sensitivity of $4.55 \times 10^{-3} \mathrm{kPa}^{-1}$. Further, the low voltage $(\sim 100 \mathrm{mV})$ operation of the presented pressure sensors makes them ideal for wearable electronic applications. (C) 2018 Author(s). All article content, except where otherwise noted, is licensed under a Creative Commons Attribution (CC BY) license (http://creativecommons.org/ licenses/by/4.0/). https://doi.org/10.1063/1.5030545
\end{abstract}

The ability to mimic the tactile functionality of human skin has become an area of immense interest owing to its potential applications in robotics, ${ }^{1-3}$ tactile internet of things (IOT), haptics, ${ }^{4}$ prosthesis, ${ }^{5}$ and wearable devices. ${ }^{6}$ Among various receptors present in the human skin, those responsible for pressure/force are the most critical, ${ }^{1}$ and as a result, pressure sensors based on various transduction mechanisms (e.g., capacitive, ${ }^{3}$ piezoresistive, ${ }^{7,8}$ and piezoelectric ${ }^{9-11}$ ) have been explored. Due to distributed nature of tactile sensing, several of these sensors are used in an array configuration as a passive or active matrix. ${ }^{12}$ Owing to the ease of integration, local processing of data, and addressing, field effect transistor (FET) based pressure sensors are attractive in such a configuration, and therefore, FET based pressure sensors have been developed by integrating a transducer material within the transistors such as in the gate or channel region. ${ }^{10,11,13-15}$ The FET based sensors are generally very sensitive, and their flexible or bendable versions have also been reported. ${ }^{12,14}$ However, they usually require highvoltage operation and are energy inefficient, which is particularly a major issue when several of these sensors are needed for large area tactile skin type applications. ${ }^{6}$ Here, we report a low voltage piezoelectric graphene field effect transistor (GFET) based pressure sensor. The device comprises GFET connected with a piezoelectric capacitor structure in an extended gate configuration. The application of pressure results in the generation of piezo-potential which modulates the channel current of GFET. Graphene is an attractive material for FETs owing to its fascinating intrinsic material properties, ${ }^{16,17}$ mechanical flexibility, and high carrier mobility which facilitate development of low voltage devices with excellent mechanical durability. ${ }^{12,14}$ Gaining from excellent properties of graphene, the reported devices will enable the development of high density low voltage sensors which are critical for emulation of human skin. In comparison to previously reported work on pressure sensors, ${ }^{3,11,18}$ the current sensors operate at much lower voltage and exhibit a higher sensitivity (Table I) which are attractive features for robotics and wearable systems.

For the fabrication of the sensing device, a CVD grown graphene on $\mathrm{Cu}$ foil (from Graphenea) was used as the channel material of GFET. The key fabrication process steps are schematically shown in Figs. 1(a)-1(f). Graphene was wettransferred onto thermally grown $\mathrm{SiO}_{2}$ (300 nm thick) on a p-type Si substrate, with cellulose acetate butyrate (CAB) acting as the support layer during the transfer process. ${ }^{19}$ During the transfer process, $\mathrm{Cu}$ foil was etched in $0.5 \mathrm{M}$ iron (III) chloride solution. The graphene/CAB stack was transferred to deionized (DI) water from the etchant. Subsequently, the graphene/CAB was transferred to a modified RCA 1 solution $\left(\mathrm{H}_{2} \mathrm{O}: \mathrm{HCl}: \mathrm{H}_{2} \mathrm{O}_{2}\right.$ 20:1:1) and one more time to the DI water before finally transferred to the $\mathrm{SiO}_{2} / \mathrm{Si}$ subsrate. ${ }^{20}$ The sample was heated at $50^{\circ} \mathrm{C}$ for $5 \mathrm{~min}$ and then baked in a $\mathrm{N}_{2}$ ambient oven at $80^{\circ} \mathrm{C}$ for $30 \mathrm{~min}$. Following this, the polymer was removed by overnight immersion in acetone. Source and drain electrodes $(10 \mathrm{~nm} /$ $40 \mathrm{~nm} \mathrm{Ti} / \mathrm{Au}$ ) of GFET were realized by photolithography, electron beam (e-beam) evaporation, and a standard lift-off process. The channel isolation of the device was achieved using a combination of photolithography and $\mathrm{O}_{2}$ plasma reactive ion etching (RIE) at $300 \mathrm{~W}$ for $13 \mathrm{~s}$. Prior to atomic layer deposition (ALD), $2 \mathrm{~nm}$ thick $\mathrm{Al}$ was deposited via ebeam evaporation which served as the nucleation layer for subsequent ALD deposition. $25 \mathrm{~nm}$ thick high-K dielectric $\mathrm{Al}_{2} \mathrm{O}_{3}$ was then deposited via thermal ALD at $200^{\circ} \mathrm{C}$ using trimethylaluminium (TMA) and $\mathrm{H}_{2} \mathrm{O}$ as precursors. The top gate dielectric capacitance of the device is $\sim 234.8 \mathrm{nF} / \mathrm{cm}^{2}$. Finally, the top gate electrode $(10 \mathrm{~nm} / 40 \mathrm{~nm} \mathrm{Ti} / \mathrm{Au})$ was defined using photolithography followed by metallization and lift-off. The vias to the source and drain electrodes was opened by a controlled etching with diluted $\mathrm{HF}\left(100: 1 \mathrm{H}_{2} \mathrm{O}\right.$ : $\mathrm{HF})$. The optical micrograph of the fabricated device is shown in Fig. 1(g). The extended gate configuration was achieved by forming an electrical contact between the top 
TABLE I. Comparison of key performance indicators of the reported pressure sensors.

\begin{tabular}{lcccc}
\hline \hline & Organic FET $(\mathrm{DNTT})^{11}$ & Silicon FET $^{10}$ & GFET $^{12}$ & GFET (This work) $^{2}$ \\
\hline Sensitivity $\left(\mathrm{GPa}^{-1}\right)$ & 1.96 & 6.12 & $\sim 205$ & 4550 \\
Operating voltage $(\mathrm{V})$ & $\mathrm{V}_{\mathrm{ds}}=\mathrm{V}_{\mathrm{gs}}=-2 \mathrm{~V}$ & $\mathrm{~V}_{\mathrm{ds}}=\mathrm{V}_{\mathrm{gs}}=5 \mathrm{~V}$ & $\mathrm{~V}_{\mathrm{ds}}=0.1 ; \mathrm{V}_{\mathrm{gs}}=25 \mathrm{~V}$ & $\mathrm{~V}_{\mathrm{ds}}=0.1 ; \mathrm{V}_{\mathrm{gs}}=0 \mathrm{~V}$ \\
Mobility $\left(\mathrm{cm}^{2} / \mathrm{V}-\mathrm{s}\right)$ & 0.56 & 696 & $\mu_{\mathrm{h}}=212, \mu_{\mathrm{e}}=96$ & $\mu_{\mathrm{h}}=879, \mu_{\mathrm{e}}=828$ \\
Pressure regime $(\mathrm{kPa})$ & $0-320$ & $0-5550$ & $0.250-3000$ & $0-94.18$ \\
\hline \hline
\end{tabular}

gate electrode and the bottom electrode of the piezoelectric metal-insulator-metal (MIM) structure of $9 \mathrm{~mm}$ in diameter as shown schematically in Fig. 1(h). The MIM structure comprises the brass substrate with lead zirconate titanate (PZT) as the piezoelectric layer and Ag as the top electrode.

The quality of transferred graphene was studied using Raman spectroscopy and atomic force microscopy (AFM). The Raman spectrum of graphene transferred using the above transfer techniques on the $\mathrm{SiO}_{2} / \mathrm{Si}$ substrate is shown in Fig. S1(a). The absence of the D-peak in Raman spectra of graphene shows a high-quality transfer. In addition to the absence of the D-peak, a single Lorentzian fit based estimation of the full width at half maximum of the $2 \mathrm{D}$ peak was $\sim 30 \mathrm{~cm}^{-1}$ and the intensity ratio between $2 \mathrm{D}$ and $\mathrm{G}$ peaks was $\sim 2$, all of which implies a high-quality transfer of monolayer graphene. The AFM characterization of graphene transferred on top of the $\mathrm{SiO}_{2} / \mathrm{Si}$ substrate is shown in Fig. S1(b). From the topography, it can be observed that the estimated average roughness corresponds to $0.9 \mathrm{~nm}$ which further confirms the high-quality transfer. The electrical characterization of GFET was carried out using a Keysight B1500A. The transfer and the output characteristics of the device are shown in Figs. 2(a) and 2(b), respectively. The (a)

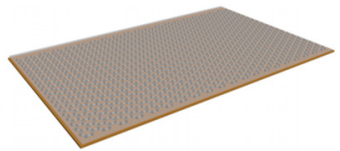

(c)

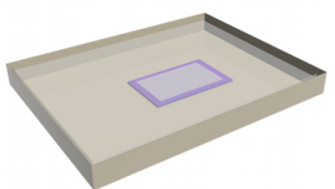

(e)

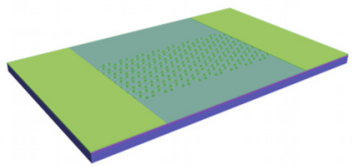

(g)

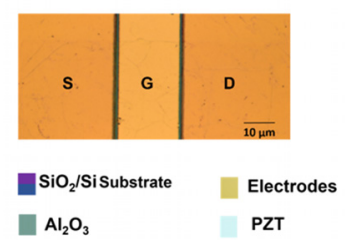

(b)

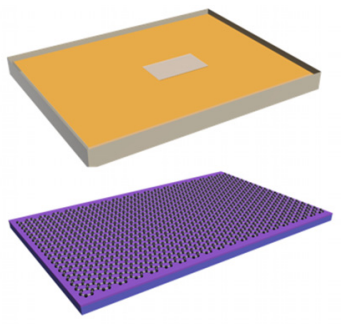

(f)

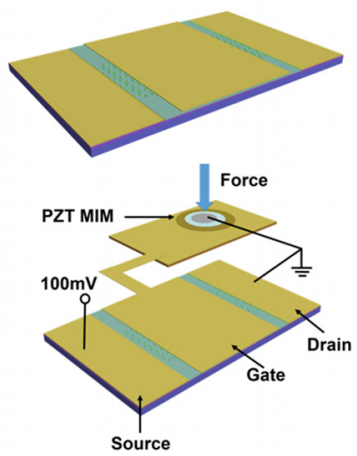

FIG. 1. Schematic representation of key fabrication steps of top gate GFET: (a) $\mathrm{CAB}$ spin-coated on graphene/Cu. (b) Etching of $\mathrm{Cu}$ in iron (III) chloride solution with $\mathrm{CAB}$ as the support layer. (c) Removal of $\mathrm{CAB}$ after the transfer of graphene onto the $\mathrm{SiO}_{2} / \mathrm{Si}$ substrate. (d) Graphene transferred to $\mathrm{SiO}_{2} /$ Si. (e) Source/ Drain electrodes and channel definition, ALD $\mathrm{Al}_{2} \mathrm{O}_{3}$ deposition. (f) Top gate Electrode deposition and opening of source/drain vias. (g) Optical Micrograph of Top Gate GFET. (h) Extended gate configuration of pressure sensitive GFET. observed shift in the Dirac point of transfer characteristics of GFET can be attributed to the unintentional doping arising from polymeric residues and ambient doping of graphene. ${ }^{21}$ The carrier mobility of GFET is determined using the diffusive transport model $^{22}$

$$
R_{\text {total }}=R_{s}+\frac{L_{G}}{W} \frac{1}{e \mu \sqrt{n_{0}^{2}+n^{2}}},
$$

where $R_{\text {total }}$ is the total GFET resistance due to the series resistance $R_{S}$ together with the gate-voltage dependent graphene channel resistance. $R_{S}$ is the series resistance arising from contact resistance and ungated region of the channel, $L_{G}$ and $W$ are the top gate channel length $(35 \mu \mathrm{m})$ and width $(50 \mu \mathrm{m})$, respectively, $e$ is the electron charge, $\mu$ is the carrier mobility, $n_{0}$ is the residual carrier density, and $n$ is the carrier density modulated by the potential on the top gate. The carrier density, $n$, is related to top gate bias by ${ }^{22}$

$$
V_{T G}-V_{T G, \text { Dirac }}=\frac{n e}{C_{o x}}+\frac{\hbar v_{f} \pi n}{e},
$$

where $v_{f}$ is the Fermi velocity $=1.15 \times 10^{6} \mathrm{~m} / \mathrm{s}^{23}$ and $V_{T G}$ $-V_{T G, \text { Dirac }}$ arises from the carrier density modulated by top gate and quantum capacitance of the graphene. The carrier mobility of holes and electrons was extracted by separate fitting for each case using Eqs. (1) and (2). A separate fitting was used due to the asymmetry in the hole and electron branches around the Dirac point. The observed asymmetry around the Dirac point arises due to doping by metal con$\operatorname{tact}^{24}$ and due to different long range scattering strengths between different charge carriers at the oxide/graphene interface, resulting in suppression of conduction of a particular carrier type. ${ }^{25}$ The fitting of the transfer curve is shown in the inset of Fig. 2(a). The extracted hole and electron carrier mobilities of GFET are $873 \mathrm{~cm}^{2} / \mathrm{Vs}$ and $830 \mathrm{~cm}^{2} / \mathrm{V} \mathrm{s}$, respectively.
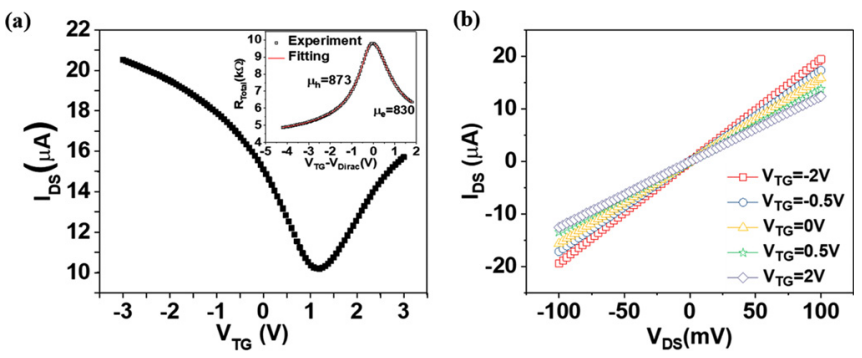

FIG. 2. (a) Electrical characteristics of GFET. (a) Transfer characteristics of GFET at $V_{\mathrm{DS}}=100 \mathrm{mV}$. The inset shows the total device resistance at $\mathrm{V}_{\mathrm{ds}}=100 \mathrm{mV}$ with respect to the Dirac voltage and equation fit (solid red line) with $\mathrm{R}_{\mathrm{s}}$ of $4077 \Omega$ and $4474 \Omega$ for hole and electron branches, respectively. (b) Output characteristics of GFET for varying top gate voltages. 
(a)

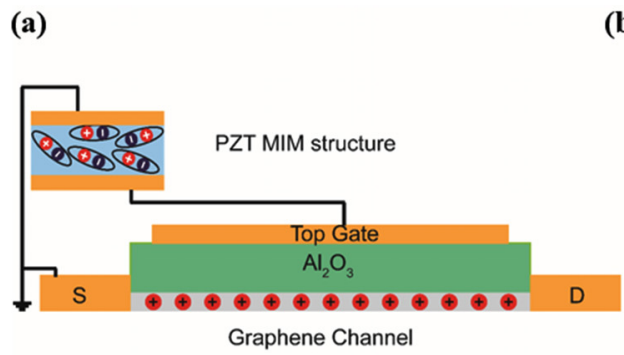

(b)

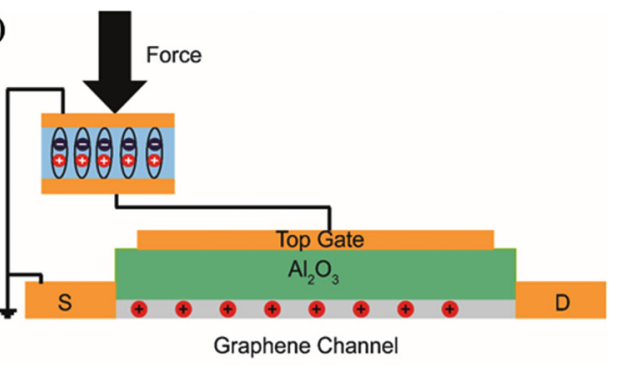

FIG. 3. Schematic representation of the pressure sensing mechanisms of the sensor. (a) In the absence of any force application. (b) Application of force results in the piezo-potential due to the alignment of dipoles within the piezoelectric MIM structure. The generated piezo-potential results in the repulsion of the like charge carriers resulting in the decrease in drain current.
Following the electrical characterization of the device, their pressure sensing performance was evaluated by applying a dynamic force of varying amplitudes using a linear stage motor controlled via a LabVIEW program. The linear stage motor's movement can be precisely controlled to $1 \mu \mathrm{m}$ resolution. This was utilized to apply a controlled force on the sensor. Prior to the application of force, calibration of the system was carried out using a load cell (RS 414 0843). The sensing mechanisms of the fabricated pressure sensor under an applied force are shown schematically in Fig. 3. The pressure sensing characteristics of the sensor could be explained as follows: The force applied to the piezoelectric transducer results in a net dipole moment within the piezoelectric material due to the non-centrosymmetric property of the material, resulting in piezo-potential generation; the generated piezopotential is applied to the top-gate electrode of GFET resulting in observed modulation of the channel current. The relationship between applied force and charge generated can be approximately described as ${ }^{1}$

$$
Q=d_{33} F,
$$

where $d_{33}$ is the piezoelectric coefficient and $\mathrm{F}$ is the applied force. As the generated charge is linearly dependent on the applied force, the generated piezo-potential is directly dependent on the magnitude of force. Thus, a higher magnitude of force/pressure results in a higher magnitude of piezopotential and hence in the larger modulation of drain current. Figure 4(a) depicts the extended gate performance under different magnitudes of pressure with the inset showing the linearity of the piezopotential generated with respect to pressure. During the pressure sensing characterization of the sensor, GFET was biased at a $V_{d s}$ of $100 \mathrm{mV}$ and with no back-gate or top gate voltage applied. An application of pressure results in the distribution of net dipole moments within PZT resulting in a positive piezo-potential which is applied to the top gate of GFET. This results in the decrease in the channel current of the device due to the repulsion of holes. Upon release of the pressure, the piezo-potential disappears resulting in an increase in charge carriers. This results in the drain current regaining its original value as shown in Fig. 4(b). The sensor response to varying magnitudes of pressure $(0-94.18 \mathrm{kPa})$ is shown in Fig. 4(b). This pressure range is similar to the pressure experience during a normal touch and object manipulation. ${ }^{1,26}$ The higher magnitude of pressure resulted in a larger modulation of the channel current, which is attributed to a higher magnitude of piezo-potential generated during the touch and release event. The sensitivity of sensor is determined using $\left(\left(\Delta \mathrm{I} / \mathrm{I}_{0}\right) / \Delta \mathrm{P}\right)$, where $\Delta \mathrm{P}$ is the
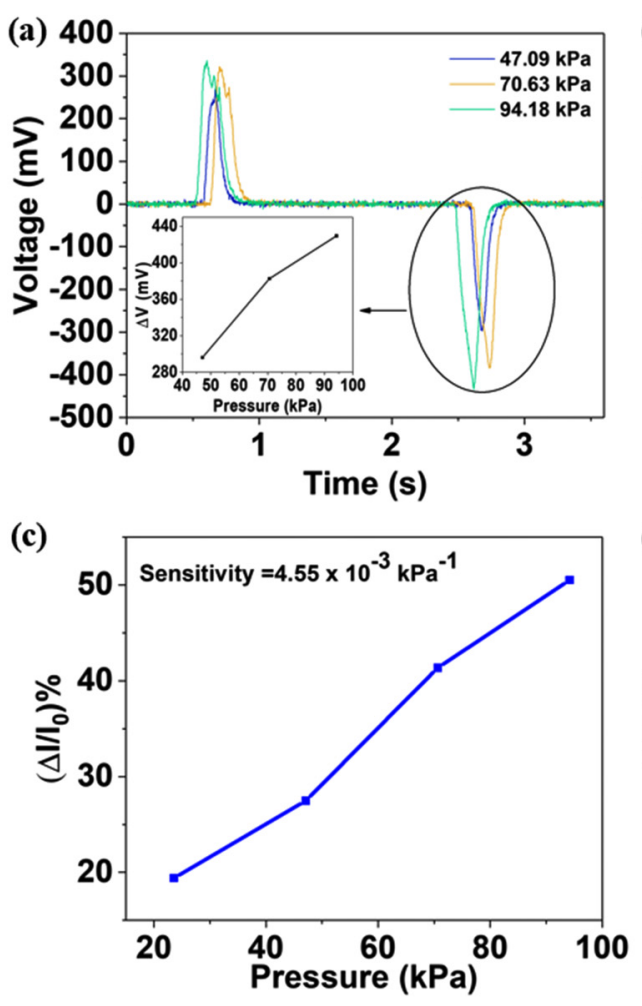
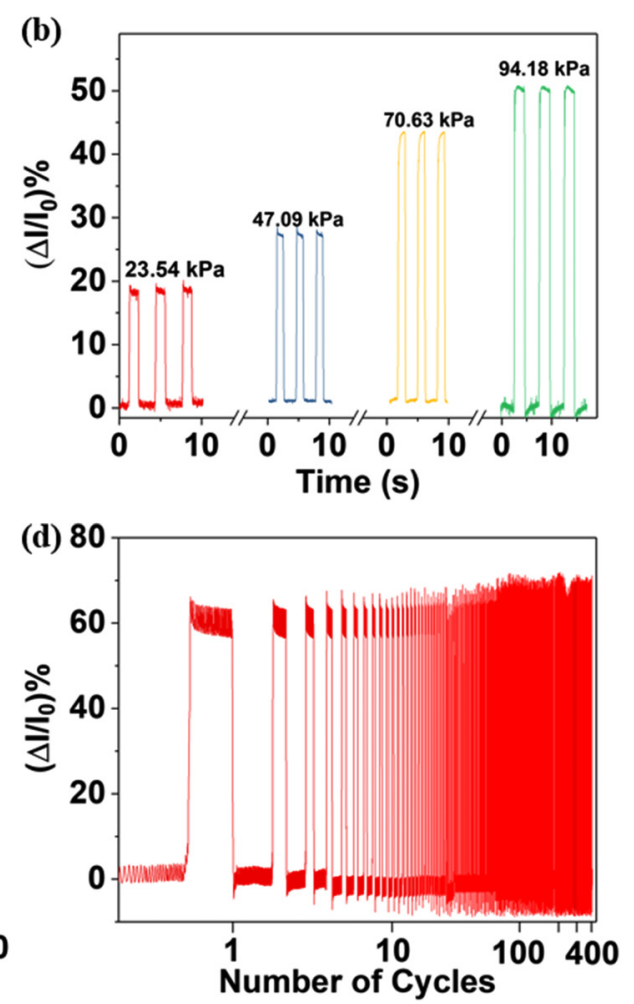

FIG. 4. (a) Pressure response of the piezoelectric capacitive structure for different pressures; the inset shows the piezopotential generated for varying applied voltages. (b) Response of GFET for varying magnitudes of pressure. (c) Normalized change in current vs pressure. (d) Cyclic measurements of sensor over 400 switching cycles for a pressure of $94.18 \mathrm{kPa}$. 
change in pressure and is calculated to be $4.55 \times 10^{-3} \mathrm{kPa}^{-1}$ for a given pressure range as shown in Fig. 4(c). The sensor sensitivity is higher than that of the other reported tactile sensors as summarized in Table I. Table I summarizes the performance merits of different sensors in comparison with this GFET based pressure sensor. Besides sensitivity and low operational voltage of the sensor, its long-term stability is another important parameter for application in real world conditions. The stability of the sensor was evaluated by performing a switching cyclic test under a pressure of $94.18 \mathrm{kPa}$. The sensor exhibited a stable performance over 400 cycles with no noticeable degradation in the performance, as shown in Fig. 4(d). In addition to the above features, the simple fabrication process of the presented sensor with respect to Si FET offers a significant advantage towards low-cost.

In summary, a piezo-potential operated extended gate GFET pressure sensor has been developed using the low temperature CMOS compatible process in combination with transfer printing. The sensor operates at a very low voltage of $100 \mathrm{mV}$, thereby enabling its potential use in applications such as wearable electronics and electronic skin. The device exhibited a sensitivity over a wide range of pressures with a sensitivity of $4.55 \times 10^{-3} \mathrm{kPa}^{-1}$, which is better than that of the Si and organic FET based sensors. In addition, the sensor exhibited a stable response over 400 cycles with no noticeable degradation in its performance during cyclic switching test, thereby exhibiting its usability for real world applications. The reported sensor architecture could be further improved by integrating the pressure sensitive piezoelectric layer within the dielectric stack of device which could be achieved by using a polymeric piezoelectric layer.

See supplementary material for the Raman spectroscopy and AFM image of transferred graphene.

This work was supported by the European Commission under Grant Agreement No. PITN-GA-2012-317488CONTEST and Engineering and Physical Science Council (EPSRC) Fellowship for Growth-Printable Tactile Skin (No. EP/M002527/1). The authors are thankful to the support received for this work from James Watt Nanofabrication Centre (JWNC).
${ }^{1}$ R. S. Dahiya and M. Valle, Robotic Tactile Sensing: Technologies and System (Springer Science \& Business Media, 2012).

${ }^{2}$ N. Yogeswaran, W. Dang, W. T. Navaraj, D. Shakthivel, S. Khan, E. O. Polat, S. Gupta, H. Heidari, M. Kaboli, L. Lorenzelli, G. Cheng, and R. Dahiya, Adv. Rob. 29(21), 1359 (2015).

${ }^{3}$ C. G. Núñez, W. T. Navaraj, E. O. Polat, and R. Dahiya, Adv. Funct. Mater. 27(18), 1606287 (2017).

${ }^{4}$ S. Bochereau, S. Sinclair, and V. Hayward, ACM Trans. Appl. Percept. 15(2), 1 (2018).

${ }^{5}$ W. Taube Navaraj, C. García Núñez, D. Shakthivel, V. Vinciguerra, F. Labeau, D. H. Gregory, and R. Dahiya, Front. Neurosci. 11, 501 (2017).

${ }^{6}$ R. Dahiya, W. T. Navaraj, S. Khan, and E. Polat, Inf. Disp. 31(4), 2-6 (2015).

${ }^{7}$ L. Pan, A. Chortos, G. Yu, Y. Wang, S. Isaacson, R. Allen, Y. Shi, R. Dauskardt, and Z. Bao, Nat. Commun. 5, 3002 (2014).

${ }^{8}$ J. S. Lee, K.-Y. Shin, O. J. Cheong, J. H. Kim, and J. Jang, Sci. Rep. 5, 7887 (2015).

${ }^{9}$ W. Wu, X. Wen, and Z. L. Wang, Science 340(6135), 952-957 (2013).

${ }^{10}$ R. S. Dahiya, A. Adami, C. Collini, and L. Lorenzelli, Sens. Actuators, A 202, 226 (2013).

${ }^{11}$ S. Hannah, A. Davidson, I. Glesk, D. Uttamchandani, R. Dahiya, and H. Gleskova, Org. Electron. 56, 170 (2018).

${ }^{12}$ S.-H. Shin, S. Ji, S. Choi, K.-H. Pyo, B. Wan An, J. Park, J. Kim, J.-Y. Kim, K.-S. Lee, S.-Y. Kwon, J. Heo, B.-G. Park, and J.-U. Park, Nat. Commun. 8, 14950 (2017).

${ }^{13}$ C.-T. Lee and Y.-S. Chiu, Appl. Phys. Lett. 106(7), 073502 (2015).

${ }^{14}$ Q. Sun, D. H. Kim, S. S. Park, N. Y. Lee, Y. Zhang, J. H. Lee, K. Cho, and J. H. Cho, Adv. Mater. 26(27), 4735 (2014).

${ }^{15}$ I. Graz, M. Kaltenbrunner, C. Keplinger, R. Schwödiauer, S. Bauer, S. P. Lacour, and S. Wagner, Appl. Phys. Lett. 89(7), 073501 (2006).

${ }^{16}$ K. S. Novoselov, A. K. Geim, S. V. Morozov, D. Jiang, Y. Zhang, S. V. Dubonos, I. V. Grigorieva, and A. A. Firsov, Science 306(5696), 666 (2004).

${ }^{17}$ K. S. Novoselov, V. I. Fal'ko, L. Colombo, P. R. Gellert, M. G. Schwab, and K. Kim, Nature 490, 192 (2012).

${ }^{18}$ R. S. Dahiya, G. Metta, M. Valle, A. Adami, and L. Lorenzelli, Appl. Phys. Lett. 95(3), 034105 (2009).

${ }^{19}$ T. Hallam, N. C. Berner, C. Yim, and G. S. Duesberg, Adv. Mater. Interfaces 1(6), 1400115 (2014).

${ }^{20}$ X. Liang, B. A. Sperling, I. Calizo, G. Cheng, C. A. Hacker, Q. Zhang, Y. Obeng, K. Yan, H. Peng, Q. Li, X. Zhu, H. Yuan, A. R. Hight Walker, Z. Liu, L.-M. Peng, and C. A. Richter, ACS Nano 5(11), 9144 (2011).

${ }^{21}$ A. D. Bartolomeo, F. Giubileo, F. Romeo, P. Sabatino, G. Carapella, L. Lemmo, T. Schroeder, and G. Lupina, Nanotechnology 26(47), 475202 (2015).

${ }^{22}$ S. Kim, J. Nah, I. Jo, D. Shahrjerdi, L. Colombo, Z. Yao, E. Tutuc, and S. K. Banerjee, Appl. Phys. Lett. 94(6), 062107 (2009).

${ }^{23}$ L. A. Ponomarenko, R. Yang, R. V. Gorbachev, P. Blake, A. S. Mayorov, K. S. Novoselov, M. I. Katsnelson, and A. K. Geim, Phys. Rev. Lett. 105(13), 136801 (2010).

${ }^{24}$ B. Huard, N. Stander, J. A. Sulpizio, and D. Goldhaber-Gordon, Phys. Rev. B 78(12), 121402 (2008).

${ }^{25}$ H. Xu, Z. Zhang, H. Xu, Z. Wang, S. Wang, and L.-M. Peng, ACS Nano 5(6), 5031-5037 (2011).

${ }^{26}$ Y. Zang, F. Zhang, C.-A. Di, and D. Zhu, Mater. Horiz. 2(2), 140-156 (2015). 